Annals of Pure and Applied Mathematics

Vol. 16, No. 1, 2018, 133-140

ISSN: 2279-087X (P), 2279-0888(online)

Published on 8 January 2018

Annals of

www.researchmathsci.org

DOI: http://dx.doi.org/10.22457/apam.v16n1a15

\title{
Gaussian Neighborhood Prime Labeling of Some Classes of Graphs and Cycles
}

\author{
Rajesh Kumar T.J and Mathew Varkey T.K \\ Department of Mathematics \\ TKM College of Engineering, Kollam, Kerala, India \\ Email: vptjrk@gmail.com and mathewvarkeytk@gmail.com
}

Received 12 December 2017; accepted 2 January 2018

\begin{abstract}
A graph $\mathrm{G}$ on $n$ vertices is said to have a neighborhood prime labeling if there exists a labeling from the vertices of $\mathrm{G}$ to the first $n$ natural numbers such that for each vertex in $\mathrm{G}$ with degree greater than one, the neighborhood vertices have relatively prime labels. Gaussian integers are the complex numbers whose real and imaginary parts are both integers. We extend the neighborhood prime labeling concept to Gaussian integers. Using the order on the Gaussian integers, we show that some classes of graphs and cycles are Gaussian neighborhood prime graphs.
\end{abstract}

Keywords: Gaussian integers, Gaussian neighborhood prime labeling

AMS Mathematics Subject Classification (2010): $05 \mathrm{C} 78$

\section{Introduction}

Graph labeling where the vertices are assigned values subject to certain conditions have many applications in Engineering and Science. One of the very appropriate application in real life problem is described in [10]. For all terminology and notations in Graph theory, we follow [1] and for all terminology regarding graph labeling, we follow [2]. A graph on $\mathrm{n}$ vertices is said to have a prime labeling if its vertices can be labeled with the first $\mathrm{n}$ natural numbers in such a way that any two adjacent vertices have relatively prime labels. In [4], Steven Klee, Hunter Lehmann and Andrew Park extend the notion of prime labeling to Gaussian integers. They define a spiral ordering on the Gaussian integers that allow us to linearly order the Gaussian integers. Steven Klee proved that the path graph, star graph, spider graph, n-centipede tree, double star tree and firecracker tree admits Gaussian prime labeling. Labeling of $(n, 2)$ centipede tree was discussed in [7].

A graph $\mathrm{G}$ on $\mathrm{n}$ vertices is said to have a neighborhood prime labeling [3] if there exists a labeling from the vertices of $G$ to the first $n$ natural numbers such that for each vertex in $\mathrm{G}$ with degree greater than one, the neighborhood vertices have relatively prime. Neighborhood prime labeling of some graphs were discussed in [5]. Kulli [8], [9] discussed the edge neighborhood graphs and neighborhood transformation of graphs. In this Paper we extend the study of neighborhood prime labeling to Gaussian integers. In section 2, we discuss the properties of spiral ordering in Gaussian integers. In section 3, we apply the properties of spiral ordering to prove the Gaussian neighborhood prime labeling of some classes of graphs and cycles. 


\section{Rajesh Kumar T.J and Mathew Varkey T.K.}

\section{Gaussian neighborhood prime labeling}

All graphs in this paper are finite undirected graphs without loops or multiple edges. We follow [4] for definition and information on the Gaussian integers. The Gaussian integers, denoted $Z[i]$, are the complex numbers of the form $a+b i$, where $a, b \in Z$ and $i^{2}=-1$. The norm of Gaussian integer $a+b i$, denoted by $N(a+b i)$, is given by $a^{2}+b^{2}$. A Gaussian integer is even if it is divisible by $1+\mathrm{i}$ and odd otherwise. A unit in the Gaussian integers is one of $\pm 1, \pm i$. An associate of a Gaussian integer $\alpha$ is $u$. $\alpha$ where $u$ is a Gaussian unit. A Gaussian integer $\rho$ is prime if its only divisors are $\pm 1, \pm i, \pm \rho$ or $\pm \rho i$. The Gaussian integers $\alpha$ and $\beta$ are relatively prime if their only common divisors are units in Z[i].

The Gaussian integers are not totally ordered. So, we use the spiral ordering of the Gaussian integers introduced by Steven Klee in [4].

Definition 1. [4] The spiral ordering of the Gaussian integers is a recursively defined ordering of the Gaussian integers. We denote the $\mathrm{n}^{\text {th }}$ Gaussian integer in the spiral ordering by $\gamma_{\mathrm{n}}$. The ordering is defined beginning with $\gamma_{1}=1$ and continuing as:

$$
\gamma_{n+1}=\left\{\begin{array}{rr}
\gamma_{n}+i, & \text { if } \operatorname{Re}\left(\gamma_{n}\right) \equiv 1(\bmod 2), \operatorname{Re}\left(\gamma_{n}\right)>\operatorname{Im}\left(\gamma_{n}\right)+1 \\
\gamma_{n}-i, & \text { if } \operatorname{Im}\left(\gamma_{n}\right) \equiv 0(\bmod 2), \operatorname{Re}\left(\gamma_{n}\right) \leq \operatorname{Im}\left(\gamma_{n}\right)+1, \operatorname{Re}\left(\gamma_{n}\right)>1 \\
\gamma_{n}+1, & \text { if } \operatorname{Im}\left(\gamma_{n}\right) \equiv 1(\bmod 2), \operatorname{Re}\left(\gamma_{n}\right)<\operatorname{Im}\left(\gamma_{n}\right)+1 \\
\gamma_{n}+i, & \text { if } \operatorname{Im}\left(\gamma_{n}\right) \equiv 0(\bmod 2), \operatorname{Re}\left(\gamma_{n}\right)=1 \\
\gamma_{n}=i, & \text { if } \operatorname{Re}\left(\gamma_{n}\right) \equiv 0(\bmod 2), \operatorname{Re}\left(\gamma_{n}\right) \geq \operatorname{Im}\left(\gamma_{n}\right)+1, \operatorname{Im}\left(\gamma_{n}\right)>0 \\
\gamma_{n}+1, & \text { if } \operatorname{Re}\left(\gamma_{n}\right) \equiv 0(\bmod 2), \operatorname{Im}\left(\gamma_{n}\right)=0 .
\end{array}\right.
$$

The first 10 Gaussian integers under this ordering are $1,1+\mathrm{i}, 2+\mathrm{i}, 2,3,3+\mathrm{i}, 3+2 \mathrm{i}, 2+2 \mathrm{i}$, $1+2 \mathrm{i}, 1+3 \mathrm{i}, \ldots \ldots$. and $\left[\gamma_{\mathrm{n}}\right]$ denote the set of the first $\mathrm{n}$ Gaussian integers in the spiral ordering. Here we exclude the imaginary axis to ensure that the spiral ordering excludes associates.

spiral ordering.

In [4] Steven Klee proved the following properties of Gaussian integers in

(1) Let $\alpha$ be a Gaussian integer and $\mathrm{u}$ is a unit. Then $\alpha$ and $\alpha+\mathrm{u}$ are relatively prime.

(2) Consecutive Gaussian integers in the spiral ordering are relatively prime.

(3) Let $\alpha$ be an odd Gaussian integer, let $\mathrm{c}$ be a positive integer, and let $\mathrm{u}$ be a unit. Then $\alpha$ and $\alpha+\mathrm{u} .(1+\mathrm{i})^{\mathrm{c}}$ are relatively prime.

(4) Consecutive odd Gaussian integers in the spiral ordering are relatively prime.

(5) Let $\alpha$ be a Gaussian integer and let $p$ be a prime Gaussian integer. Then $\alpha$ and $\alpha+p$ are relatively prime if and only if $\mathrm{p}$ does not divides $\alpha$.

Now we define the neighborhood prime labeling with Gaussian integers using the definition of the spiral ordering for Gaussian integers. For a vertex $v \in V(G)$, the neighborhood of $\mathrm{v}$ is the set of all vertices in $\mathrm{G}$ which are adjacent to $\mathrm{v}$ and is denoted by $\mathrm{N}(\mathrm{v})$.

Definition 2. [4] Let $\mathrm{G}$ be a graph on $\mathrm{n}$ vertices. A Gaussian neighborhood prime labeling of $G$ is a bijection $f: V(G) \rightarrow\left[\gamma_{n}\right]$ such that for each vertex $v \in V(G)$ with $\operatorname{deg}(v)$ $>1,\left\{\mathrm{f}\left(\mathrm{u}_{\mathrm{i}}\right): \mathrm{u}_{\mathrm{i}} \in \mathrm{N}(\mathrm{v})\right\}$ are relatively prime. A graph which admits Gaussian neighborhood prime labeling is called Gaussian neighborhood prime graph. 
Gaussian neighborhood prime labeling of some classes of graphs and cycles

\section{Gaussian neighborhood prime labeling of some graphs}

Patel and Shrimali [3] introduced neighborhood prime labeling and the discussion of the labeling on some graphs is in [5]. Gaussian neighborhood prime labeling of some graphs were discussed in [6]. We now discuss the Gaussian neighborhood prime labeling for some graphs using the properties of spiral ordering in the Gaussian integers.

Definition 3. Let $\mathrm{p}, \mathrm{n}, \mathrm{m}, \mathrm{n} \leq \mathrm{m}$ be integers. The (p, n, m) double star tree is the union of two star graph $\mathrm{K}_{1, \mathrm{n}}$ and $\mathrm{K}_{1, \mathrm{~m}}$ together with a path of length $\mathrm{p}$ joining their apex vertices.

Theorem 1. Any (p, n, m) double star tree is a Gaussian neighborhood prime graph.

Proof: Let $\mathrm{G}$ be a $(\mathrm{p}, \mathrm{n}, \mathrm{m})$ double star tree which is the union of two star graphs $\mathrm{K}_{1, \mathrm{n}}$ and $\mathrm{K}_{1, \mathrm{~m}} \quad(\mathrm{n} \leq \mathrm{m})$. Let $\mathrm{v}_{1}, \mathrm{v}_{2}, \mathrm{v}_{3}, \ldots, \mathrm{v}_{\mathrm{p}}$ be the vertices of the path $\mathrm{P}_{\mathrm{p}}$ which joins the star graphs $K_{1, n}$ and $K_{1, m}$ where $v_{1}$ and $v_{p}$ are the apex vertices of them. The pendant vertices of $K_{1, n}$ are $\mathrm{v}_{\mathrm{p}+1}, \mathrm{v}_{\mathrm{p}+2}, \ldots, \mathrm{v}_{\mathrm{p}+\mathrm{n}}$ and the pendant vertices of $\mathrm{K}_{1, \mathrm{~m}}$ are $\mathrm{v}_{\mathrm{p}+\mathrm{n}+1}, \mathrm{v}_{\mathrm{p}+\mathrm{n}+2}, \ldots, \mathrm{v}_{\mathrm{p}+\mathrm{n}+\mathrm{m}}$. Label the vertex $\mathrm{v}_{1}$ with $\gamma_{2}=1+\mathrm{i}$ and label the vertex $\mathrm{v}_{\mathrm{p}}$ with $\gamma_{1}=1$. Label the remaining $\mathrm{p}-1$ vertices of the path $\mathrm{P}_{\mathrm{n}}$ by defining the labeling $\mathrm{f:V}\left(\mathrm{P}_{\mathrm{p}}\right) \rightarrow\left\{\gamma_{3}, \gamma_{4}, \ldots, \gamma_{\mathrm{p}}\right\}$ as

Case (1): if $\mathrm{p}$ is even

Case (2): if p is odd

$$
\begin{aligned}
& f\left(v_{2 i)}\right)=\gamma_{\left(\frac{p}{2}+i+1\right)}, 1 \leq i \leq \frac{p-2}{2} \\
& f\left(v_{(2 i+1)}=\gamma_{(i+2)}, 1 \leq i \leq \frac{p-2}{2}\right.
\end{aligned}
$$

$$
\begin{aligned}
& f\left(v_{2 i)}\right)=\gamma_{\left(\frac{p-1}{2}+i+1\right)}, 1 \leq i \leq \frac{p-1}{2} \\
& f\left(v_{(2 i+1)}=\gamma_{(i+2)}, 1 \leq i<\frac{p-1}{2}\right.
\end{aligned}
$$

The labeling on the path is a Gaussian neighborhood prime labeling since the neighborhood vertices are consecutive Gaussian integers. Now, label the pendent vertices $\mathrm{v}_{\mathrm{p}+1}, \mathrm{v}_{\mathrm{p}+2}, . ., \mathrm{v}_{\mathrm{p}+\mathrm{n}}$ of the star graph $\mathrm{K}_{1, \mathrm{n}}$ with the remaining $\mathrm{n}$ odd Gaussian integers. Label the pendant vertices in $K_{1, m}$ with the remaining $m$ Gaussian integers. Then, $G$ admits a Gaussian neighborhood prime labeling.

Definition 4. A comb graph is a caterpillar in which each vertex in the path is joined to exactly one pendent vertex and is denoted by $\mathrm{P}_{\mathrm{n}} \odot \mathrm{K}_{1}$.

Theorem 2. Any comb graph $\mathrm{P}_{\mathrm{n}} \odot \mathrm{OK}_{1}, \mathrm{n} \in \mathrm{N}$ is a Gaussian neighborhood prime graph.

Proof: Let $v_{1}, v_{2}, \ldots, v_{n}$ are the vertices in the path $P_{n}$ and $u_{1}, u_{2}, \ldots, u_{n}$ are the pendant vertices of the comb graph $P_{n} \odot K_{1}$. The pendent vertex $u_{n}$ is labeled with $\gamma_{2}=1+i$ and the remaining pendant vertices are labeled with $\mathrm{n}-1$ remaining even Gaussian integers. The vertices in the path are labeled with odd Gaussian integers by defining the labeling $\mathrm{f}: \mathrm{V}$ $\left(\mathrm{P}_{\mathrm{n}} \odot \mathrm{K}_{1}\right) \rightarrow\left\{\gamma_{1}, \gamma_{3} \ldots \gamma_{2 \mathrm{n}-1}\right\}$ as

Case (1) if $n$ is even

$$
f\left(v_{(2 i-1)}\right)=\gamma_{\left(\frac{2 n-2}{2}+2 i\right)}, 1 \leq i \leq \frac{n}{2}
$$


Rajesh Kumar T.J and Mathew Varkey T.K.

Case (2) if $\mathrm{n}$ is odd

$$
f\left(v_{2 i}\right)=\gamma_{(2 i-1)}, 1 \leq i \leq \frac{n}{2}
$$

$$
\begin{aligned}
& f\left(v_{(2 i-1)}=\gamma_{\left(\frac{2 n-4}{2}+2 i\right)}, 1 \leq i \leq \frac{n+1}{2}\right. \\
& f\left(v_{2 i}\right)=\gamma_{(2 i-1)}, 1 \leq i \leq \frac{n-1}{2}
\end{aligned}
$$

The labeling shows that adjacent vertices of every vertex in the path except $\mathrm{v}_{1}$ and $\mathrm{v}_{\mathrm{n}}$ are consecutive odd Gaussian integers and they are relatively prime. The adjacent vertices of $\mathrm{v}_{1}$ contains the Gaussian integer $\gamma_{1}=1$ which is relatively prime to all the Gaussian integers. The adjacent vertices of $\mathrm{v}_{\mathrm{n}}$ contains the Gaussian integer $\gamma_{2}=1+\mathrm{i}$ which is relatively prime to all the odd Gaussian integers. So the labeling is a Gaussian neighborhood prime labeling for the comb graph.

Definition 5. A spider graph is a tree in which one vertex is of degree at least 3 called the center of the spider and all other vertices having degree at most 2. A leg of a spider is a path from center to a vertex of degree one. In a spider graph $S_{m}, m \in N$ there are $m$ paths $\mathrm{P}_{1}, \mathrm{P}_{2}, . ., \mathrm{P}_{\mathrm{m}}$ attached to the center vertex.

Theorem 3. A spider graph $S_{m}$ is a Gaussian neighborhood prime graph.

Proof: Let $\mathrm{v}$ be the center vertex of the spider graph $\mathrm{S}_{\mathrm{m}}$ which has degree $\mathrm{m}$. Then if we remove $v$ from $S_{m}$ we are left with $m$ paths $P_{1}, P_{2}, \ldots, P_{m}$ of lengths $1_{1}, 1_{2}, \ldots, 1_{m}$. Label the vertex $v$ with Gaussian integer $\gamma_{1}=1$. Let $v_{1}, v_{2}, \ldots, v_{l_{1}}$ are the vertices of the path $P_{1}$. We now label the vertices of $\mathrm{P}_{1}$ with the following labeling.

Case (1) if $l_{1}$ is even

Case (2) if $1_{1}$ is odd

$$
\begin{aligned}
& f\left(v_{(2 i-1)}\right)=\gamma_{(i+1)}, 1 \leq i \leq \frac{l_{1}}{2} \\
& f\left(v_{2 i}\right)=\gamma_{\left(\frac{l_{1}}{2}+i+1\right)}, 1 \leq i \leq \frac{l_{1}}{2}
\end{aligned}
$$

$$
\begin{aligned}
& f\left(v_{(2 i-1)}\right)=\gamma_{(i+1)}, \quad 1 \leq i \leq \frac{l_{1}+1}{2} \\
& f\left(v_{2 i}\right)=\gamma_{\left(\frac{l_{1}+1}{2}+i+1\right)}, \quad 1 \leq i \leq \frac{l_{1}-1}{2}
\end{aligned}
$$

Let $\mathrm{v}_{1}, \mathrm{v}_{2} \ldots, v_{l_{2}}$ are the vertices of the path $\mathrm{P}_{2}$. Label the vertices of path $\mathrm{P}_{2}$ with the following labeling

Case (1) if $l_{2}$ is even

Case (2) if $l_{2}$ is odd

$$
\begin{gathered}
f\left(v_{(2 i-1)}\right)=\gamma_{\left(l_{1}+i+1\right)}, 1 \leq i \leq \frac{l_{2}}{2} \\
f\left(v_{2 i}\right)=\gamma_{\left(\frac{l_{2}}{2}+l_{1}+i+1\right)}, 1 \leq i \leq \frac{l_{2}}{2}
\end{gathered}
$$

$$
f\left(v_{(2 i-1)}\right)=\gamma_{\left(l_{1}+i+1\right)}, \quad 1 \leq i \leq \frac{l_{2}+1}{2}
$$


Gaussian neighborhood prime labeling of some classes of graphs and cycles

and so on.

$$
f\left(v_{2 i}\right)=\gamma_{\left(\frac{l_{2}+1}{2}+l_{1}+i+1\right)}, \quad 1 \leq i \leq \frac{l_{2}-1}{2}
$$

In generally, the labeling on the arbitrary path $P_{s}$ of length $l_{s}$ if the previous path $P_{t}$ has length $1_{t}$ is

Case (1) if $1_{\mathrm{s}}$ is even

Case (2) if $1_{\mathrm{S}}$ is odd

$$
\begin{aligned}
& f\left(v_{(2 i-1)}\right)=\gamma_{\left(l_{t}+i+1\right)}, 1 \leq i \leq \frac{l_{s}}{2} \\
& \mathrm{f}\left(\mathrm{v}_{2 \mathrm{i}}\right)=\gamma_{\left(\frac{\mathrm{s}_{s}}{2}+\mathrm{l}_{\mathrm{t}}+\mathrm{i}+1\right)}, 1 \leq \mathrm{i} \leq \frac{\mathrm{l}_{s}}{2}
\end{aligned}
$$

$$
\begin{aligned}
& f\left(v_{(2 i-1)}\right)=\gamma_{\left(l_{t}+i+1\right)}, \quad 1 \leq i \leq \frac{l_{s}+1}{2} \\
& f\left(v_{2 i}\right)=\gamma_{\left(\frac{l_{s}+1}{2}+l_{t}+i+1\right)}, \quad 1 \leq i \leq \frac{l_{s}-1}{2}
\end{aligned}
$$

From the above labeling, it is to be clear that, the labeling on the neighborhood vertices of all the vertices in the paths have consecutive Gaussian integers. So spider graph $S_{m}$ admits Gaussian neighborhood prime labeling.

Definition 6. The $(\mathrm{n}, 2)$-centipede tree, $\mathrm{C}_{\mathrm{n}, 2}$ is the graph with $\mathrm{V}\left(\mathrm{c}_{\mathrm{n}, 2}\right)=\left\{\mathrm{v}_{1}, \mathrm{v}_{2}, \ldots, \mathrm{v}_{3 \mathrm{n}}\right\}$, and $\mathrm{E}\left(\mathrm{c}_{\mathrm{n}, 2}\right)=\left\{\mathrm{v}_{3 \mathrm{k}-1} \mathrm{v}_{3 \mathrm{k}-2}, \mathrm{v}_{3 \mathrm{k}-1} \mathrm{v}_{3 \mathrm{k}}: 1 \leq \mathrm{k} \leq \mathrm{n}\right\} \mathrm{U}\left\{\mathrm{v}_{3 \mathrm{k}-1} \mathrm{v}_{3 \mathrm{k}+2}: 1 \leq \mathrm{k} \leq \mathrm{n}-1\right\} . \mathrm{C}_{\mathrm{n}, 2}$ has $\mathrm{n}$ vertices on its spine with indices that are congruent to $2(\bmod 3)$ and each vertex on the spine has two pendant vertices adjacent to it. We call each spine vertex $v_{3 k-1}$ and its neighboring vertices $\mathrm{v}_{3 \mathrm{k}-2}$ and $\mathrm{v}_{3 \mathrm{k}}$ the $\mathrm{k}^{\text {th }}$ segment of the tree.

Theorem 4. Any (n, 2) - centipede tree is a Gaussian neighborhood prime graph.

Proof: In the spine of the tree $\mathrm{C}_{\mathrm{n}, 2}$ we set the vertices are $\left\{v_{3 k-1}\right\}_{k=1}^{n}$ which have degree greater than one and the other vertices are pendent vertices. The vertices in the spine are $\left\{\mathrm{v}_{2}, \mathrm{v}_{5}, \ldots, \mathrm{v}_{6 \mathrm{k}+5}, \ldots\right\}$. The spine vertices are the only vertices which have degree greater than one in $C_{n, 2}$. Label the vertices $v_{j}$ with $\gamma_{j}$. In a same segment each spine leaf pair will be labeled with consecutive Gaussian integers. But the labeling on the neighborhood vertices of the spine vertex is not relatively prime. Now, we will swap the labels of the spine vertex with labels of one of their pendant vertices. We swap each $\gamma_{3 \mathrm{k}-1}$ on the spine with its neighboring pendent vertex $\gamma_{3 k}$.In the new labeling, the pendant vertices in each segment have labeled with consecutive Gaussian integers. In each segment where a swap occurred the labeling on neighborhood vertices of the spine vertex have consecutive Gaussian integers. Then, $\mathrm{C}_{\mathrm{n}, 2}$ admits a Gaussian neighborhood prime labeling.

Theorem 5. Every cycle $C_{n}$ if $n ¥ 2(\bmod 4)$ is a Gaussian neighborhood prime graph.

Proof: Let $v_{1}, v_{2}, \ldots, v_{n}$ be the vertices of the cycle $C_{n}$. Consider the labeling $f: V\left(C_{n}\right) \rightarrow$ $\left[\gamma_{\mathrm{n}}\right]$ as follows

Case (1): If $\mathrm{n}$ is odd

$$
\begin{aligned}
& f\left(v_{2 j-1}\right)=\gamma_{\left(\frac{n-1}{2}\right)+j}, 1 \leq j \leq \frac{n+1}{2} \\
& f\left(v_{2 j}\right)=\gamma_{j}, 1 \leq j \leq \frac{n-1}{2}
\end{aligned}
$$




\section{Rajesh Kumar T.J and Mathew Varkey T.K.}

The neighborhood vertices of each vertex in the labeling have consecutive Gaussian integers. Consecutive Gaussian integers in the spiral ordering are relatively prime. So $C_{n}$ if $\mathrm{n}$ is odd and $\mathrm{n} ¥ 2(\bmod 4)$ admits Gaussian neighborhood prime labeling.

Case (2): If $\mathrm{n}$ is even

$$
\begin{aligned}
& f\left(v_{2 j-1}\right)=\gamma_{\frac{n}{2}+j}, 1 \leq j \leq \frac{n}{2} \\
& f\left(v_{2 j}\right)=\gamma_{j}, 1 \leq j \leq \frac{n}{2}
\end{aligned}
$$

The neighborhood vertices of each vertex in the labeling have consecutive Gaussian integers except the vertex $v_{n}$. The labeling on the neighborhood vertices of $v_{n}$ are $\gamma_{\left(\frac{n}{2}+1\right)}$ and $\gamma_{n}$. If $n \neq 2(\bmod 4)$ the difference of the labeling on the neighborhood vertices in spiral ordering are prime Gaussian integers. Then using property (5) in spiral ordering of Gaussian integers $\gamma_{\left(\frac{n}{2}+1\right)}$ and $\gamma_{n}$ are relatively prime. Therefore the above labeling is Gaussian neighborhood prime labeling for the cycle $\mathrm{C}_{\mathrm{n}}$ if $\mathrm{n} \neq 2(\bmod 4)$.

Theorem 6. Every cycle $C_{n}$ with a chord is Gaussian neighborhood prime for $n \geq 4$.

Proof: Let $\mathrm{G}$ be a graph joining two non-adjacent vertices of cycle $\mathrm{C}_{\mathrm{n}}$ with a chord. Let $v_{1}, v_{2}, \ldots, v_{n}$ be the vertices of $G$ and $v_{i}$ and $v_{j}$ are the vertices joined by a chord . If $n \neq$ 2 (mod 4) choose the same labeling as in above theorem. Then $G$ is a Gaussian neighborhood prime. Now consider the case when $n \cong 2(\bmod 4)$ and choose the same labelling as in above theorem. Then there exists at least one vertex whose neighbourhood set is not relatively prime. Let $v_{i}$ be the vertex whose neighborhood set is not relatively prime. Now join a chord with the vertex $\mathrm{v}_{\mathrm{j}}$ which is relatively prime to the neighborhood set in $v_{i}$. Then $G$ is Gaussian neighborhood prime.

Corollary 1. Every cycle $C_{n}$ with $n-3$ chords from a vertex is Gaussian neighborhood prime for $\mathrm{n} \geq 5$.

Proof: Let $v_{1}, v_{2}, \ldots, v_{n}$ be the vertices of the cycle $C_{n}$. Choose an arbitrary vertex $v_{i}$ and joining $v_{i}$ to all the vertices which are not adjacent to $v_{i}$. Then there are n-3 chords from $v_{i}$ and the above theorem shows that it is Gaussian neighborhood prime.

Definition 7. A vertex switching $G_{v}^{\prime}$ of a graph $\mathrm{G}$ is obtained by taking a vertex v of $\mathrm{G}$, removing all the edges incident with it and adding edges joining it to every verte $\mathrm{x}$ which are not adjacent to $\mathrm{G}$.

Theorem 7. The graph obtained by switching of any vertex in a cycle $C_{n}$ is Gaussian neighborhood prime graph.

Proof: Let $G_{v}^{\prime}$ be the graph obtained by switching the vertex v of the cycle $\mathrm{C}_{\mathrm{n}}$. Then $\mathrm{v}$ is adjacent to all the $\mathrm{n}-3$ vertices in $\mathrm{C}_{\mathrm{n}}$. Now, label the vertex $\mathrm{v}$ with $\gamma_{1}=1$ and label the remaining $\mathrm{n}-1$ vertices with the Gaussian integers $\gamma_{2}, \gamma_{3}, \gamma_{4}, . ., \gamma_{\mathrm{n}}$. Then labeling on the neighborhood vertices of every vertex with degree greater than one except the vertex $\mathrm{v}$ contains $\gamma_{1}=1$. Since 1 is relatively prime to all the Gaussian integers, the labeling is Gaussian neighborhood prime. 
Gaussian neighborhood prime labeling of some classes of graphs and cycles

Definition 8. The duplication of a vertex $v$ of a graph $G$ produces a new graph $G_{v}$ by adding a vertex $v^{\prime}$ with $\mathrm{N}(\mathrm{v})=\mathrm{N}\left(v^{\prime}\right)$. In other words a vertex $v^{\prime}$ is said to be a duplication of $\mathrm{v}$ if all the vertices which are adjacent to $\mathrm{v}$ are now adjacent to $v^{\prime}$.

Theorem 9. The graph obtained by duplicating arbitrary vertex of a cycle $C_{n}$ is a Gaussian neighborhood prime graph.

Proof: Let $v_{1}, v_{2}, \ldots, v_{n}$ are the vertices of the cycle $C_{n}$. Let $G$ be the graph obtained by duplicating the vertex $\mathrm{v}_{1}$ and $v_{1}^{\prime}$ be its duplicated vertex. Then there exists an edge joining $v_{1}^{\prime}$ to $\mathrm{v}_{2}$ and $v_{1}^{\prime}$ to $\mathrm{v}_{\mathrm{n}}$. Consider the labeling $\mathrm{f}: \mathrm{V}\left(\mathrm{C}_{\mathrm{n}}\right) \rightarrow\left[\gamma_{\mathrm{n}}\right]$ as follows

Case (1): If $\mathrm{n}$ is odd

Case (2): If $\mathrm{n}$ is even

$$
\begin{aligned}
& f\left(v_{2 j-1}\right)=\gamma_{\left(\frac{n-1}{2}\right)+j}, 1 \leq j \leq \frac{n+1}{2} \\
& f\left(v_{2 j}\right)=\gamma_{j}, 1 \leq j \leq \frac{n-1}{2} \\
& f\left(v_{1}^{\prime}\right)=\gamma_{n+1}
\end{aligned}
$$

$$
\begin{aligned}
& f\left(v_{2 j-1}\right)=\gamma_{\frac{n}{2}+j}, 1 \leq j \leq \frac{n}{2} \\
& f\left(v_{2 j}\right)=\gamma_{j}, 1 \leq j \leq \frac{n}{2} \\
& f\left(v_{1}^{\prime}\right)=\gamma_{n+1}
\end{aligned}
$$

The labeling on the neighborhood vertices of every vertex in $\mathrm{G}$ except $\mathrm{v}_{1}$ and $v_{1}^{\prime}$ have consecutive Gaussian integers. The neighbourhood vertices of $\mathrm{v}_{1}$ and $v_{1}^{\prime}$ contains the Gaussian integer $\gamma_{1}=1$. Therefore $\mathrm{G}$ is Gaussian neighbourhood prime.

\section{Conclusion}

In this paper, we discussed the Gaussian neighborhood prime labeling of (p,n,m) double star tree, $(n, 2)$ centipede tree, comb graph, spider graph and cycles. The labeling on more classes of graphs will be discussed on forthcoming papers.

Acknowledgement. The authors are thankful to the referee for their valuable suggestions and helpful comments in writing this manuscript.

\section{REFERENCES}

1. F.Harary, Graph Theory, Addison Wesley, Reading, M.A.1969.

2. J.A.Gallian, A dynamic survey of graph labeling, The Electronic Journal of Combinatorics, 17(2016).

3. S.K.Patel and N.P.Shrimali, Neighborhood prime labeling, International Journal of Mathematics and Soft Computing, 5(2) (2015) 135-143.

4. S.Klee, H.Lehmann and A.Park, Prime labeling of families of trees with Gaussian integers, AKCE International Journal of Graphs and Combinatorics, 13(2) (2016) 165-176.

5. T.K.Mathew Varkey and T.J.Rajesh Kumar, A note on neighborhood prime labeling, International Journal of Mathematical Combinatorics, 4 (2016) 161-167.

6. T.J.Rajesh Kumar and T.K.Mathew Varkey, Gaussian neighborhood prime labeling of some graphs, to appear in Aryabhatta Journal of Mathematics and Informatics. 
Rajesh Kumar T.J and Mathew Varkey T.K.

7. B.S.Sunoj and T.K.Mathew Varkey, ADCSS-labeling for some middle graphs, Annals of Pure and Applied Mathematics, 12(2) (2016) 161-167.

8. V.R.Kulli, On edge neighborhood graphs, Annals of Pure and Applied Mathematics, 11(1) (2016) 79-83.

9. V.R.Kulli, On neighborhood transformation graphs, Annals of Pure and Applied Mathematics, 10(2) (2015) 239-245.

10. A.Saha, M.Pal and T.K.Pal, Selection of programme slots of television channels for giving advertisement: A graph theoretic approach, Information Sciences, 177 (12) (2007) 2480-2492 